\title{
Correction: Polarized cell migration induces cancer type-specific CD133/integrin/Src/Akt/GSK3 $\beta / \beta$-catenin signaling required for maintenance of cancer stem cell properties
}

\section{Ying-Jhen Su, Wei-Hsin Lin, Yi-Wen Chang, Kuo-Chen Wei, Chi-Lung Liang, Shin- Cheh Chen and Jia-Lin Lee}

\begin{abstract}
Present: Due to an error made during submission of the revised manuscript, Dr. Chi-Jung Liang's name was spelled incorrectly.

Corrected: The correct author list for this manuscript is provided below. Authors sincerely apologize for this oversight.
\end{abstract}

Original article: Oncotarget. 2015; 6(35): 38029-45. doi: 10.18632/oncotarget.5703.

\footnotetext{
Ying-Jhen Su ${ }^{1, *}$, Wei-Hsin Lin ${ }^{1,2, *}$, Yi-Wen Chang ${ }^{1, *}{ }^{,}$Kuo-Chen Wei $^{3}$, Chi-Jung Liang $^{1}$, Shin-Cheh Chen ${ }^{4}$ and Jia-Lin Lee ${ }^{1,5}$

${ }^{1}$ Institute of Molecular and Cellular Biology, National Tsing Hua University, Hsinchu, Taiwan

2 Department of Orthopedics, National Taiwan University Hospital Hsin-Chu Branch, Hsinchu, Taiwan

${ }^{3}$ Department of Neurosurgery, Chang-Gung Memorial Hospital, Linkou, Taiwan

${ }^{4}$ Department of Surgery, Chang-Gung Memorial Hospital, Linkou, Taiwan

${ }^{5}$ Department of Medical Science, National Tsing Hua University, Hsinchu, Taiwan

${ }^{*}$ These authors have contributed equally to this work
} 